\title{
Measurement of the Spin-Dependent Observables in Elastic NN Collisions at the NICA collider
}

\author{
Vasily Sharov* \\ Joint Institute for Nuclear Research, \\ Veksler and Baldin Laboratory of High Energy Physics \\ E-mail: vsharov41dmail.ru
}

\begin{abstract}
The report shows the possibilities of studying the spin-dependent observables in the elastic $N N$ interactions at the NICA collider of the VBLHEP JINR. On the nucleon beams of the collider it will be possible to carry out the measurements of the differential cross sections $I_{\text {oooo }}(N N)$ of the elastic scattering of unpolarized nucleons, the analyzing powers $A_{\text {oono }}(N N)$ or $A_{\text {ooon }}(N N)$ in collisions of the polarized nucleon beams with the unpolirized ones, and the parameters of the spin correlations $A_{o o k k}(N N)$ or $A_{o o n n}(N N)$ in collisions of longitudinally $(k)$ or transversely $(n)$ polarized nucleons. The planned luminosity of the colliding polarized nucleon beams will allow getting sufficiently high elastic events counting rate. The use of the colliding beams of the polarized nucleons for the $N N$ observables research has a number of significant advantages in comparison with the experiments with the "fixed" target. Firstly, the angular acceptance of the collider detector covers the full solid angle $4 \pi$ radians. Secondly, wide ranges of the investigated energies of the interacting nucleons $T_{N L a b}$ and the four-momentum transfer squared $t_{1,3}$ are available. And, finally, the so-called "target" in such measurements does not contain background impurities, which complicate the processing of accumulated data.
\end{abstract}

XXII International Baldin Seminar on High Energy Physics Problems,

15-20 September 2014

JINR, Dubna, Russia

${ }^{*}$ Speaker. 


\section{Introduction.}

One of the main physical tasks for the NICA collider will be the research in the spin particle physics [四, [D], which continues the JINR research programme in this area. The NICA collider will provide the colliding beams of polarized protons and deuterons with the energy range of $T_{p C M}=$ $5 \div 12.6 \mathrm{GeV}\left(\sqrt{s_{p p}}=12 \div 27 \mathrm{GeV}\right)$ for protons and $T_{d C M}=2 \div 5.5 \mathrm{GeV} / u\left(\sqrt{s_{N N}}=4 \div 13 \mathrm{GeV}\right)$ for deuterons. Obtaining new data on the spin-dependent observables in the elastic $N N$ scattering at the $T_{N L a b}$ energies above $1 \mathrm{GeV}$ can be one of the priority areas of $V B L H E P$ research at the collider. What kind of spin-dependent observables can be measured on the colliding $N N$ beams of the NICA collider and whether the experimental conditions on these beams are sufficiently acceptable for these measurements? The consideration of these and other issues related to the measurement of the spin $N N$ observables, is the content of this contribution.

Why is the problem of obtaining new data on the spin observables in the elastic $N N$ scattering in the region above $1 \mathrm{GeV}$ is of current importance today? It is obvious that the urgency of this problem is connected with the current state of our understanding of all aspects of the nucleonnucleon interactions. "The theory of the nucleon-nucleon $(N N)$ interaction in the range of about $1 \div$ $10 \mathrm{GeV}$ is one of the most pressing open questions of modern nuclear / particle physics. Below that energy range, chiral effective field theory applies as well as meson theory. At very high energies $(\sim 100 \mathrm{GeV})$ perturbative QCD can be used. But it is the "intermediate" region of a few $\mathrm{GeV}$ where theory has big problems. Meaningful theoretical work cannot be done unless we have also data in that critical region. The data for $n p$ are very scarce, too few to even pin down a reasonable phenomenology. For our understanding of the fundamental $N N$ interaction on a broad scale, it is vital to have data for $n p$ spin observables in the $\mathrm{GeV}$ region. These data will be useful for the entire international nuclear/particle physics Community" [B]].

At present the whole set of experimental data on the spin observables in the elastic $N N$ scattering at energies above a few $\mathrm{GeV}$ is quite poor, especially the set of $n p$ data. A complete phenomenological description of the $N N$ interactions, energy dependent phase shift analysis of the existing sets of experimental $p p$ and $n p$ data, is now possible only up to $3 \mathrm{GeV}$ for $p p$ and up to $1.3 \mathrm{GeV}$ for $n p$ collisions [ [ $⿴ 囗$ ]. There is no any dynamic theory (meson exchange, nonperturbative QCD, and so on), which could satisfactorily describe the behavior of the measured $N N$ spin-dependent observables in the area above a few $\mathrm{GeV}$. "For the past 30 years $Q C D$-based calculations have continued to disagree with the ZGS 2-spin and AGS 1-spin elastic data and the ZGS, AGS, Fermilab and RHIC inclusive data. These large spin effects do not go to zero at high energy or high- $P_{\perp}$ as was predicted. ... These precise spin experiments provide experimental guidance for the required modification of the theory of Strong Interactions" [5].

It is in the elastic $N N$ scattering that the measurements of the energy and angular behavior of the spin-dependent observables are the most preferred for the experimental studies of spin physics of the nucleon-nucleon interactions. Let us note the most attractive features of such measurements from the point of view of the experiment.

1. One of the attractive features of the experimental studies of the elastic $N N$ collisions is the fact that a complete model independent description of the scattering of the arbitrary spin particles has been elaborated very well in the relativistic quantum theory. The $S$ matrix formalism defines all possible observables in the elastic $N N$ collisions (see section [2), and all characteristics of the interaction are determined by the structure of the matrix elements - i.e., dynamic models. The experimental data on the spin-dependent elastic $N N$ observables contain sufficient information on the properties of the $N N$ interaction. Namely, the $p p$ data contain the information about an isovector $I=1$ part of the $N N$ interaction and the $n p$ data on both the isovector part $I=1$ and an isoscalar 
part $I=0$.

2. It is well known that the total elastic cross section $\sigma_{\text {totelastic }}(N N)$ of the nucleon-nucleon interaction remains large enough $\sim 7 \div 10 \mathrm{mb}$ until the $\mathrm{TeV}$ energy of the colliding nucleons. This fact will provide an acceptable count rate of the events of the elastic $N N$ collisions even for large quantities of the square four momentum transfer. Later we will present the count rate estimations of the elastic $N N$ events over the energy range of the colliding nucleons and four-momentum transfer squared available at the NICA collider.

3. The spin dependent $N N$ observables studies were performed in the collisions of the accelerated beam nucleons and nucleons in cryogenic targets using either the polarized beam or the polarized target or both the polarized beam and target. These experiments showed that some spin effects remain quite large in a wide energy range of the interacting nucleons and the square of the four momentum transfer $t$. Taken from a detailed review [6, 1993] the compilation of results on the analyzing power $A_{\text {oono }}(p p)$ for the elastic $p p$ scattering in a wide energy region up to

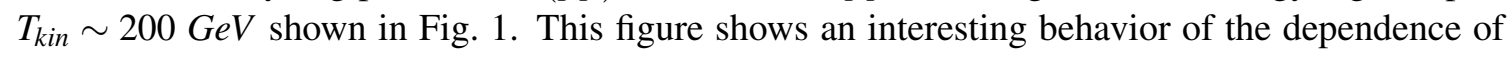

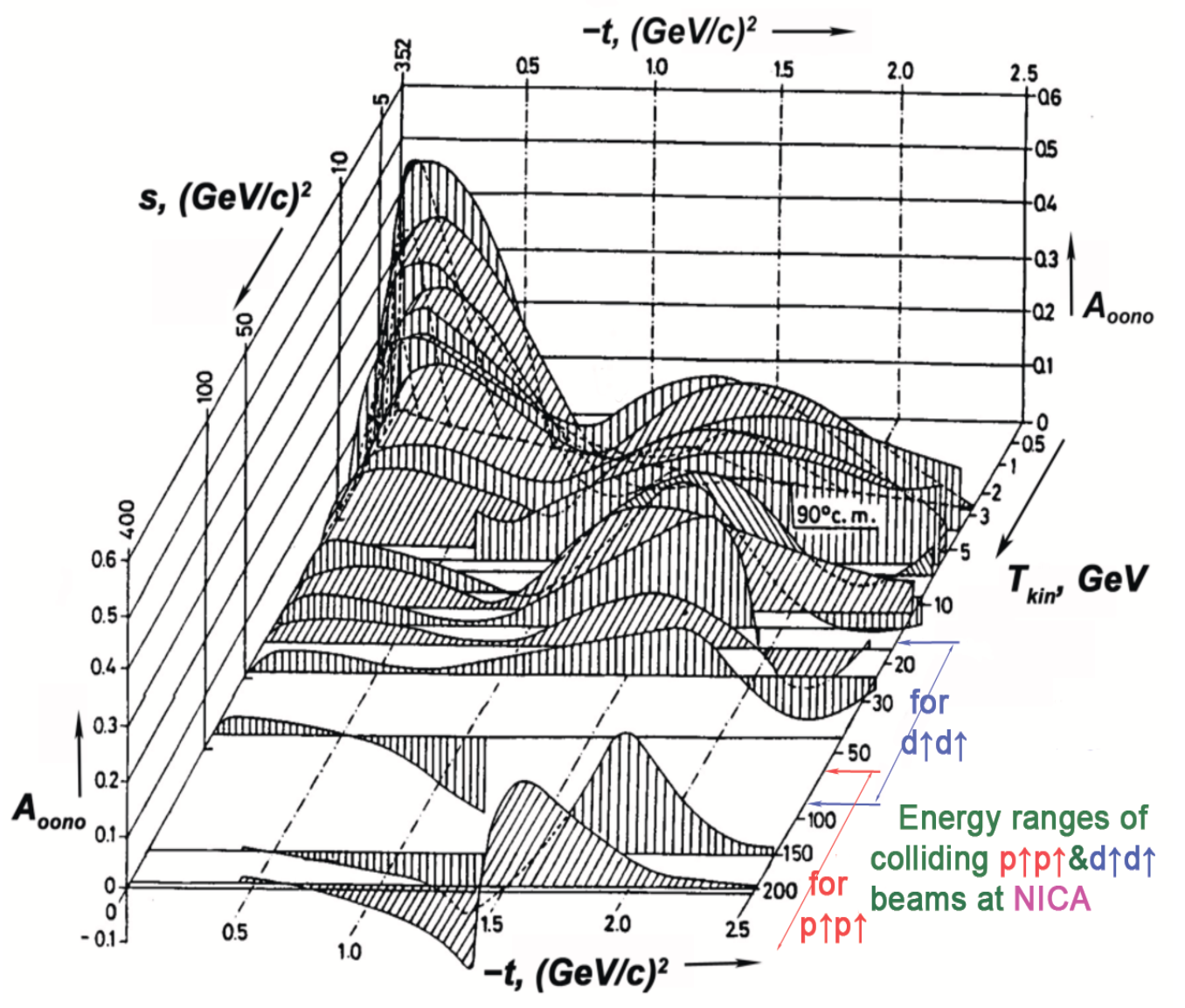

Figure 1: Analysing power $A_{o o n o}\left(T_{p L a b}, t_{1,3}\right)$ in elastic $p p$ scattering. Compilation from review [四, (1993)].

the $A_{\text {oono }}(\mathrm{pp})$ observable from the $-t$ value. A minimum at $t=\sim 1.0(\mathrm{GeV} / \mathrm{c})^{2}$ with the following maximum at $t=\sim 1.7(\mathrm{GeV} / \mathrm{c})^{2}$ are observed in all energy range. The existing $N N$ interaction dynamic models cannot explain this fact.

The $A_{\text {oono }}(p p)$ data set close to the modern one and taken from the report [ [, 2013$]$ is shown in Fig. \. Fig. \is similar to Fig. Wpublished in 1993. In both figures the red and blue arrows mark the 


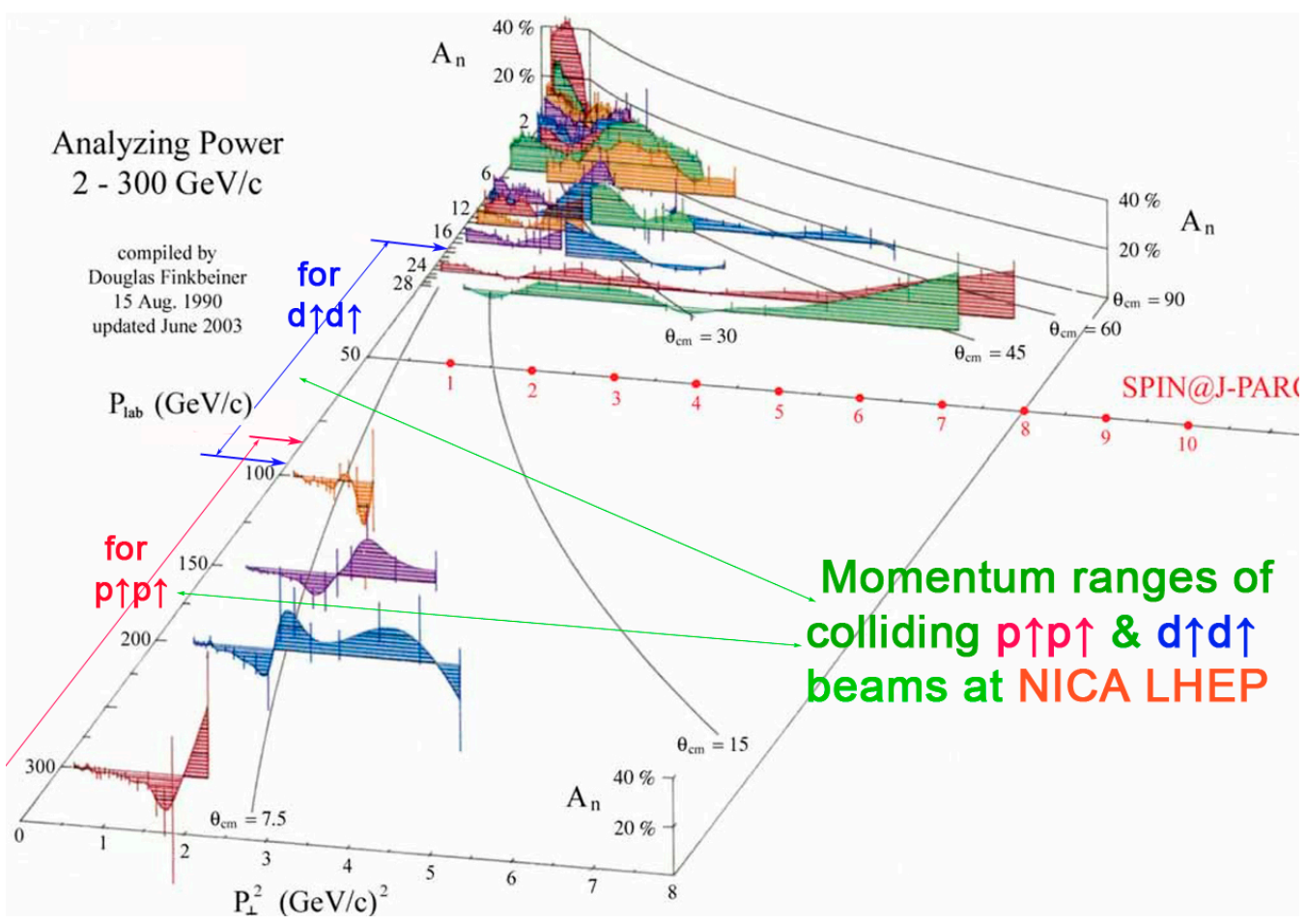

Figure 2: Similar compilation of the existed $A_{o o n o}\left(p_{p L a b}, p_{\perp}^{2}\right)$ data from [प, 2013].

planned equivalent $L a b$ energy or momentum ranges (equivalent to the "fixed" target experiment) of the colliding $p \uparrow p \uparrow$ and $d \uparrow d \uparrow$ beams of the NICA collider.

The data on the double-spin $N N$ observables $A_{\text {oonn }}$ or $A_{\text {ookk }}$ can be obtained in the experiments with the use of both the polarized nucleon beam and the polarized nucleon target or the colliding beams of polarized nucleons. As an example, Figure 3 shows the angular dependencies of the $A_{\text {oonn }}(n p)$ and $A_{\text {ookk }}(n p)$ observables measured at the energy of the incident polarized neutrons $T_{n} \sim 0.8 \mathrm{GeV}$ (see in [焑]). The existing $A_{\text {oonn }}(N N)$ and $A_{\text {ookk }}(N N)$ data sets (see for example ref's

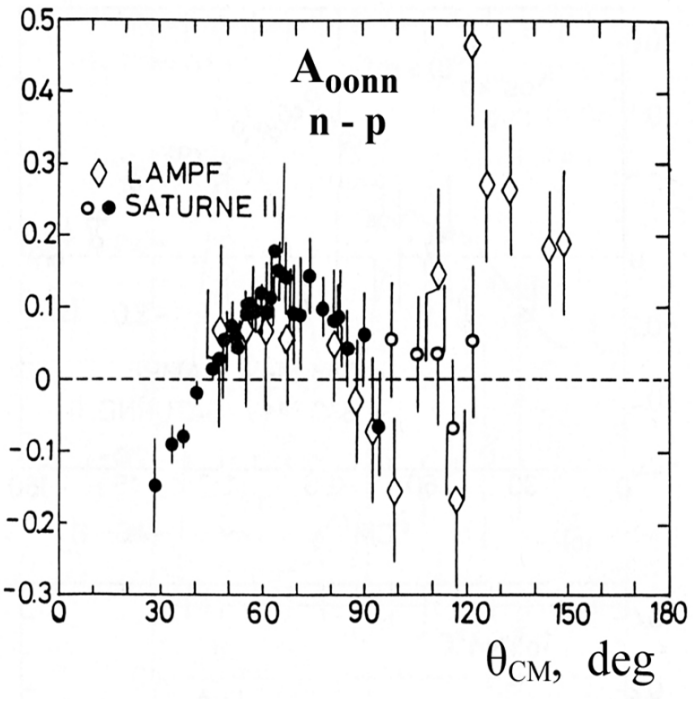

(a)

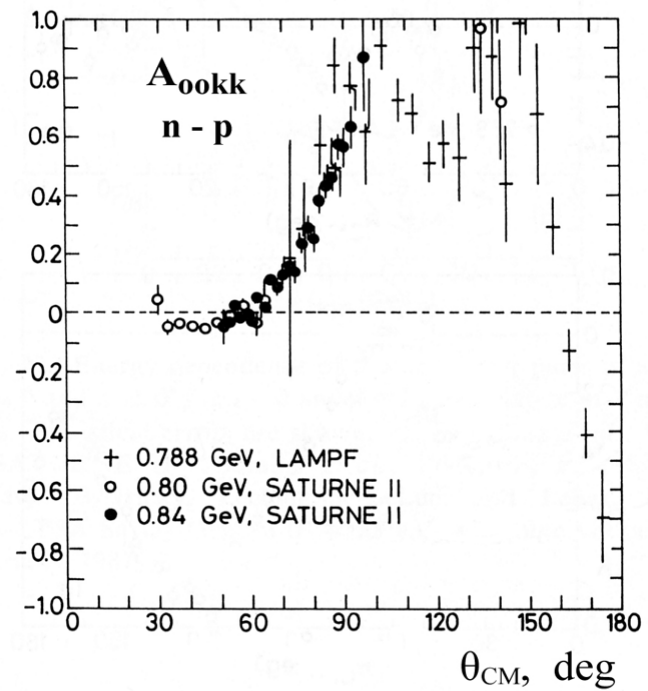

(b)

Figure 3: Spin-dependent neutron-proton elastic scattering observables. (a) $A_{\text {oonn }}(\mathrm{np})$ angular dependence around $0.8 \mathrm{GeV}$. (b) $A_{\text {ookk }}(n \mathrm{p})$ angular dependence at $\sim 0.8 \mathrm{GeV}$. 
in [6]) are quite poor, so getting such data, in particular at the NICA colliding polarized beams, is very important.

Now the spin-dependent $N N$ observables studies are ongoing in a number of the research centers and planned in some others. The first results of the studies of the elastic scattering of polarized high-energy protons have been recently obtained in the $p p 2 p p$ experiment at the RHIC collider $[8]$. The slope parameter $b$ of the diffraction peak of the elastic $p p$ cross section in the t-range of $0.010 \leq|t| \leq 0.019(\mathrm{GeV} / \mathrm{c})^{2}\left[\right.$ [Q] , the one-spin analyzing power $A_{\text {oono }}(\mathrm{pp})$ in the range of the four-momentum transfer squared $t$ of $0.01 \leq|t| \leq 0.03(\mathrm{GeV} / \mathrm{c})^{2}$ [ए]], and the double spin asymmetries $A_{\text {oonn }}(p p)$ and $A_{\text {ooss }}(p p)$ [प]] were measured with the use of the polarized proton beams of the relativistic heavy-ion collider $(R H I C)$ at proton energy of $\sqrt{s_{p}}=200 \mathrm{GeV}$.

A proposal for continuation of the nucleon spin structure research at the Spin Physics Detector (SPD) of the NICA collider has been recently presented [ए]] at VBLHEP JINR. We propose to include the measurement of the spin observables in the elastic $N N$ scattering in a research program on the $S P D$ in the energy ranges of the colliding beams of both polarized protons and polarized deuterons available at the NICA collider. The data planned to be obtained in the measurements of the elastic $N N$ scattering observables will promote a creation of an adequate phenomenological and theoretical description of the $N N$ interaction over the colliding nucleon energy $T_{N C M}$ region available at the NICA collider.

A brief definition of the spin-dependent observables in the elastic $N N$ scattering and which spin-dependent elastic $N N$ observable can be measured using the NICA detectors will be presented in the second section. The comparison of some kinematical characteristics of the nucleon-nucleon collisions at the center-of-mass $(C M)$ and laboratory $(L a b)$ systems will be done in the third section. The expected parameters of the polarized colliding $p \uparrow p \uparrow$ and $d \uparrow d \uparrow$ beams and the experimental conditions for the elastic $N N$ scattering investigation at the NICA collider will be presented in the fourth section of the article. The evaluation outputs of the elastic $N N$ events throughout the energy region of the colliding beams of the NICA collider and the comparison of the outputs of events in the experiments at the collider and in the measurements with the "fixed" target will be given in the fifth section. The sixth part of this article will provide a brief conclusion.

\section{Spin-dependent observables in the elastic NN scattering.}

In this contribution we used the $N N$ formalism and notations for the elastic nucleon-nucleon

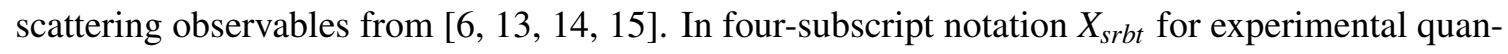
tities, introduced by [15]], subscripts $s, r, b$, and $t$ refer to the polarization components of the scattered, recoil, beam, and target particles, respectively. If an initial particle is unpolarized or a final particle polarization is not analyzed, the corresponding subscript is set equal to zero.

In theory, 256 experimental quantities exist, which can be defined as components of various tensors. Due to the symmetry principles there remain only 25 linearly independent experimental quantities. For any $C M$ observable $X_{p q i k}$, the following expression holds:

$$
d \sigma / d \Omega X_{p q i k}=\frac{1}{4} \operatorname{Tr}\left(\sigma_{1 p} \sigma_{2 q} M \sigma_{1 i} \sigma_{2 k} M^{+}\right),
$$

where $d \sigma / d \Omega=I_{\text {oooo }}=\frac{1}{4} \operatorname{Tr}\left(M M^{+}\right)$is the unpolarized differential cross section. For example, some of the $C M$ experimental quantities are listed below in term of the five invariant scattering amplitudes $a, b, c, d$, and $e$ [6, [13]].

Differential cross section:

Polarizations and analyzing powers:

$$
\begin{aligned}
& \sigma \equiv d \sigma / d \Omega=\frac{1}{2}\left(|a|^{2}+|b|^{2}+|c|^{2}+|d|^{2}+|e|^{2}\right) . \\
& \sigma P_{\text {nоoо }}=\sigma P_{\text {onoo }}=\sigma A_{\text {oоno }}=\sigma A_{\text {ooon }}=\Re e a * e .
\end{aligned}
$$


Spin correlations:

Spin correlations:

Spin correlations:

Spin correlations:

$$
\begin{aligned}
& \sigma A_{\text {oonn }}=\frac{1}{2}\left(|a|^{2}-|b|^{2}-|c|^{2}+|d|^{2}+|e|^{2}\right), \\
& \sigma A_{\text {ookk }}=-\mathfrak{R} e a * d \cos \theta+\mathfrak{R} e b * c+\mathfrak{I} m d * e \sin \theta, \\
& \sigma A_{\text {ooss }}=\mathfrak{R} e a * d \cos \theta+\mathfrak{R} e b * c+\mathfrak{I} m d * e \sin \theta, \text { and } \\
& \sigma A_{\text {oosk }}=-\mathfrak{R} e a * d \sin \theta-\mathfrak{I} m d * e \cos \theta .
\end{aligned}
$$

Which the spin-dependent elastic NN observables can be measured using the NICA detectors? A compact design of a complex detector for the colliders does not allow the scattered and recoil particles polarizations measurements. For these purposes the secondary scattering of an analyzed particle and large enough devices (polarimeters) are needed. Therefore the detectors for NICA allow measuring the following elastic $N N$ observables: the unpolarized differential $N N$ cross section $I_{\text {oooo }}$, the analyzing powers for primary reactions with the polarized beam $A_{\text {oono }}, A_{\text {ooko }}$ or target $A_{\text {ooon }}, A_{\text {oook }}$ and the spin correlation parameters $A_{\text {oonn }}$ or $A_{\text {ook }}$ in primary reactions with the beam and target both polarized transverse $n$ or longitudinally $k$.

What experimental quantities should be measured to obtain the spin-dependent NN observables? The expression for the differential cross sections for scattering of the polarized nucleon beam with energy $E$ on the polarized target nucleons with the scattered particle detection at an angle of $\theta$ is [焑]:

$$
\begin{aligned}
d \sigma / d \Omega^{p o l}(E, \theta)= & d \sigma / d \Omega^{o}(E, \theta)\left[1+A_{\text {oono }}(E, \theta) P B_{n}+A_{\text {ooon }}(E, \theta) P T_{n}+\right. \\
& +A_{\text {oonn }}(E, \theta) P B_{n} P T_{n}+A_{\text {ooss }}(E, \theta) P B_{s} P T_{s}+A_{\text {ook }}(E, \theta) P B_{k} P T_{k}+ \\
& +A_{\text {oosk }}(E, \theta)\left(P B_{s} P T_{k}+P B_{k} P T_{s}\right),
\end{aligned}
$$

where $d \sigma / d \Omega^{o}(E, \theta)$ is the cross section for unpolarized nucleons, and $P B_{k}$ and $P T_{k}$ are the polarization values of the colliding beams.

Using this formula one can obtain the expression for an asymmetry $\mathbf{a}$ in the $N N$ scattering with the opposite signs of the nucleon beam polarizations. For example, for $P B_{n}=P T_{n}=P B_{s}=P T_{s}=0$ and $P B_{k} \neq 0, P T_{k} \neq 0$ and sign $P B_{k}=1$ we have

$$
\begin{aligned}
& {[d \sigma / d \Omega]^{+}-[d \sigma / d \Omega]^{-}=2[d \sigma / d \Omega]^{o} A_{o o k k}\left|P B_{k}\right| P T_{k} \text { and }} \\
& {[d \sigma / d \Omega]^{+}+[d \sigma / d \Omega]^{-}=2[d \sigma / d \Omega]^{o},}
\end{aligned}
$$

and the asymmetry will be

$$
\mathbf{a}=\left([d \sigma / d \Omega]^{+}-[d \sigma / d \Omega]^{-}\right) /\left([d \sigma / d \Omega]^{+}+[d \sigma / d \Omega]^{-}\right)=A_{o o k k}\left|P B_{k}\right| P T_{k} .
$$

Thus in order to obtain the values of the spin-dependent $N N$ observable we have to measure the asymmetry a, that is ratio of the difference and sum of the elastic $N N$ differential cross sections for the opposite signs of the polarizations of the colliding polarized particles. To find the appropriate $d \sigma / d \Omega^{+,-, 0}(E, \theta)$ values it is necessary to measure the angular distributions of the elastically scattered $(+,-, 0)$-polarized nucleons and to normalize them to the counts of the monitor detectors of the colliding beam intensities.

If we have a possibility for extrapolation of the measured differential cross section to zero $C M$ angle, then we can obtain the spin-dependent total cross sections differences $\Delta \sigma_{L, T}(N N)$ over the NICA collider beams energy region. However, with the increasing energy of the colliding nucleons $T_{N L a b} \geq 2 \mathrm{GeV}$ the value of $\Delta \sigma_{L}(n p)$ quickly falls to zero [ए6]. This is also confirmed by the results of $E-704$ experiment at FermiLab [ए]]. 


\section{The $\mathbf{N N}$ kinematics at the center-of-mass $(C M)$ and laboratory $(L a b)$ systems.}

For completeness of the information on this issue, in addition to the above formalism of the elastic $N N$ scattering, we briefly recall some features of the elastic $N N$ kinematics in the laboratory frame $(L a b)$ and in the center of mass $(C M)$ of the colliding nucleons. The dependence of the four-momentum transfer squared $t_{1,3}$ from the beam nucleon energy $E_{N L a b}$ and from this nucleon scattering angle $\theta_{L a b}$ on the nucleon target in "normal" experiments on the elastic collisions of the beam nucleons with the "fixed" target nucleons (laboratory coordinate system) is shown in a three-dimensional Figure 由.

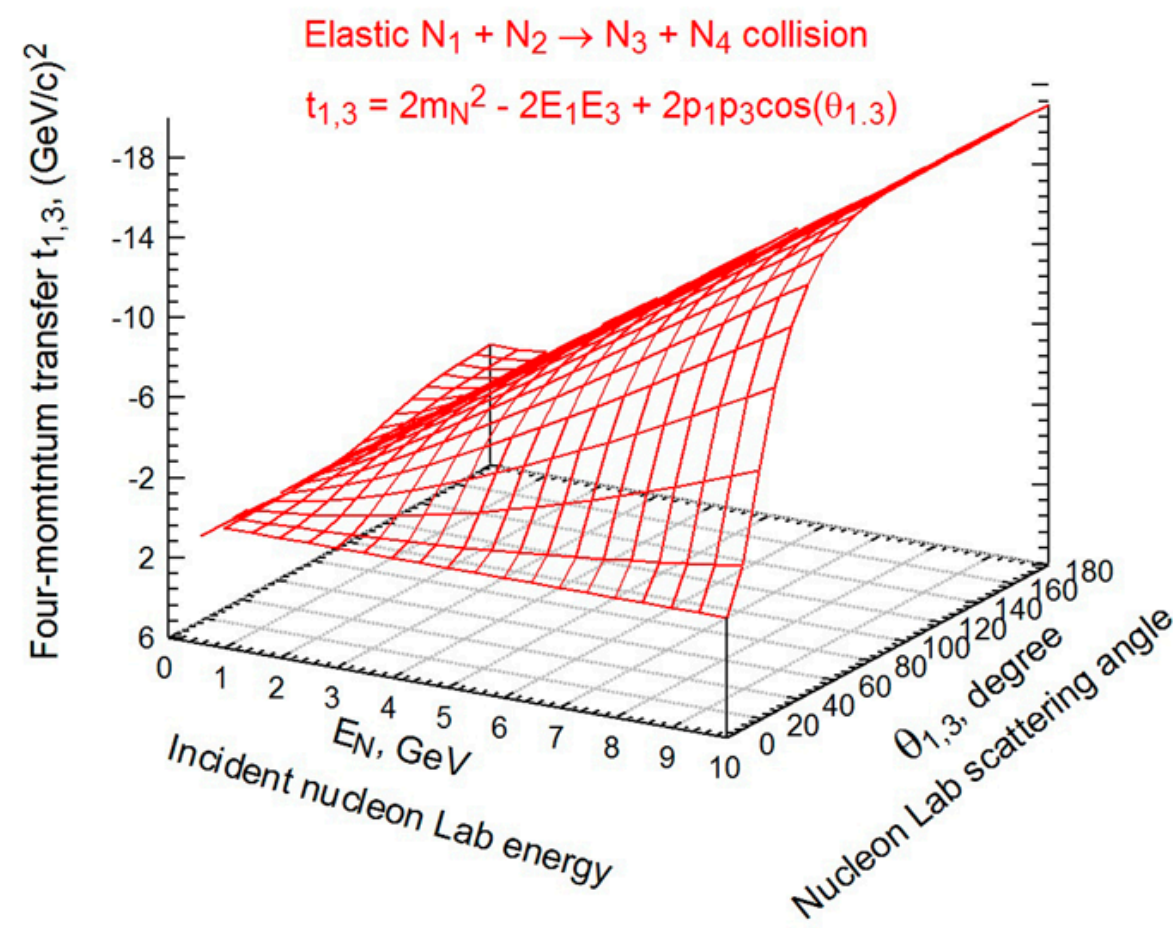

Figure 4: Kinematics of the nucleon-nucleon collisions at the laboratory $(L a b)$ system.

A similar $t_{1,3}$ dependence on the nucleon energy $E_{N C M}$ in the colliding beams and $C M$ scattering angle $\theta_{C M}$ for the elastic nucleon interactions in the colliding nucleon beams (the centre-ofmass colliding nucleons) is shown in a Fig. [1.

For the collisions in the laboratory system of coordinates Fig. $\mathbb{t}$ there is almost a linear dependence of the $t_{1,3}$ value from the energy $E_{N L a b}$ of the incident nucleon at a fixed angle of its scattering. At the energy of the incident beam nucleon $E_{N L a b} \simeq 10 \mathrm{GeV}$ and its scattering angle $\theta_{\text {Lab }} \simeq 90^{\circ}$ the four-momentum transfer squared reaches $t_{1,3} \simeq-17(\mathrm{GeV} / \mathrm{C})^{2}$. For the elastic $N N$ collisions at a centre-of-mass (Figure [D) there is a quadratic dependence of the $t_{1,3}$ value on the nucleon energy $E_{N C M}$ in the colliding beams at any $C M$ angle $\theta_{L a b}$ of its scattering. In this case, at the energy of the colliding nucleons $E_{N C M} \simeq 10 \mathrm{GeV}$ and its scattering angle $\theta_{C M} \simeq 90^{\circ}$ the square four-momentum transfer reaches $t_{1,3} \simeq-200(\mathrm{GeV} / \mathrm{c})^{2}$. That is, for the nucleon elastic collisions in the colliding beams the available area of the square four-momentum transfer is more than an order of magnitude greater than for the collisions of beam particles with the same energy in experiments with the "fixed" targets. 


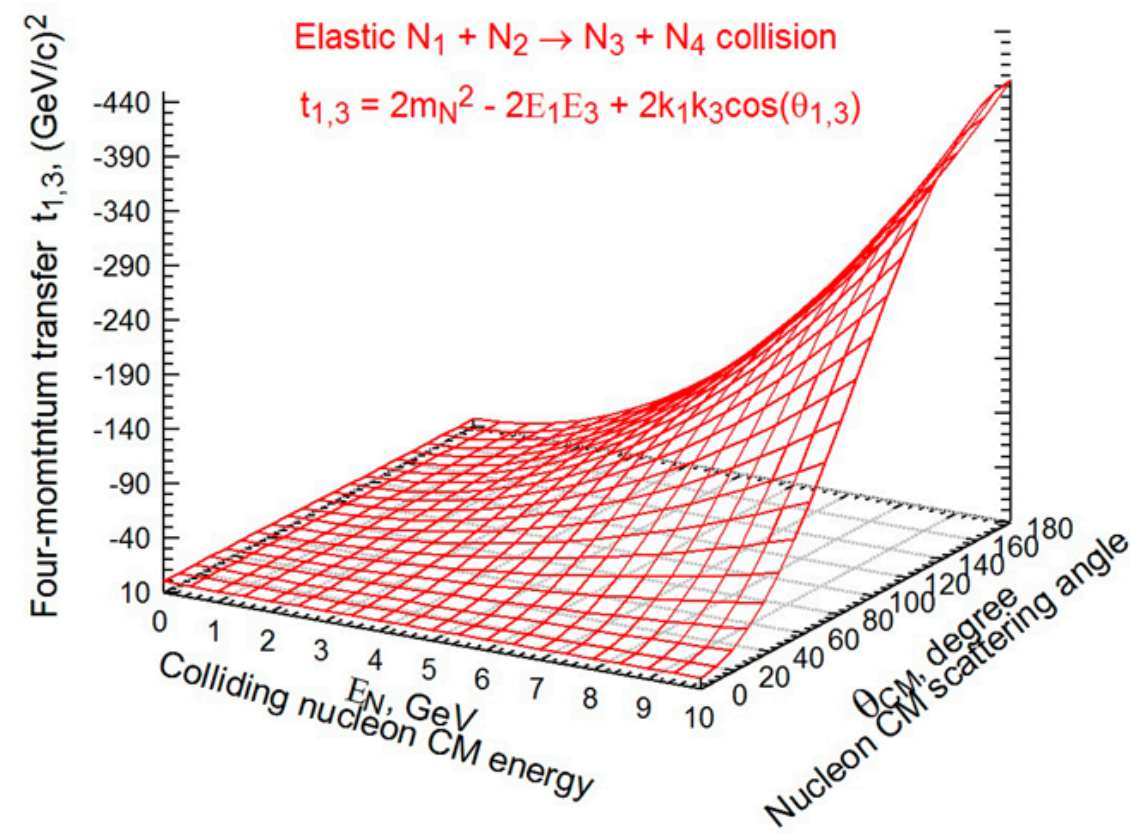

Figure 5: Kinematics of the nucleon-nucleon collisions at the center-of-mass $(C M)$ system.

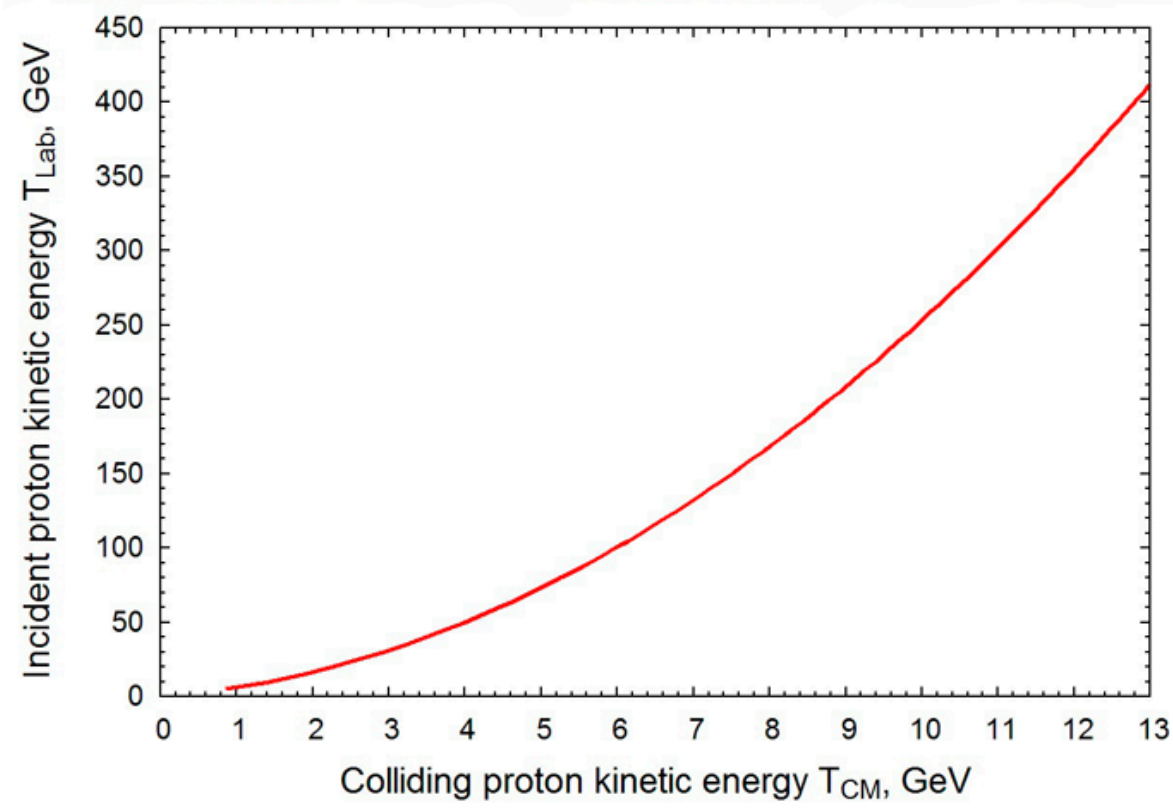

Figure 6: Incident nucleon energy $T_{N L a b}$ versus colliding nucleon one $T_{N C M}$.

Here it is also necessary to remember that the relationship between the energy $T_{N L a b}$ of the beam nucleon incident on the "fixed" target and the corresponding nucleon energy $T_{N C M}$ at the center of mass of the colliding nucleons is a complex nonlinear function. The relationship between these values is demonstrated by Fig. 6 . This figure shows that the value of the nucleon energy in the center of mass $T_{N C M} \simeq 10 \mathrm{GeV}$ corresponds to the nucleon energy in the laboratory system $T_{N L a b} \simeq 255 \mathrm{GeV}$. This is one of the significant advantages of the experiment at the collider in comparison with the experiment with neutron beam and the "fixed" target. 


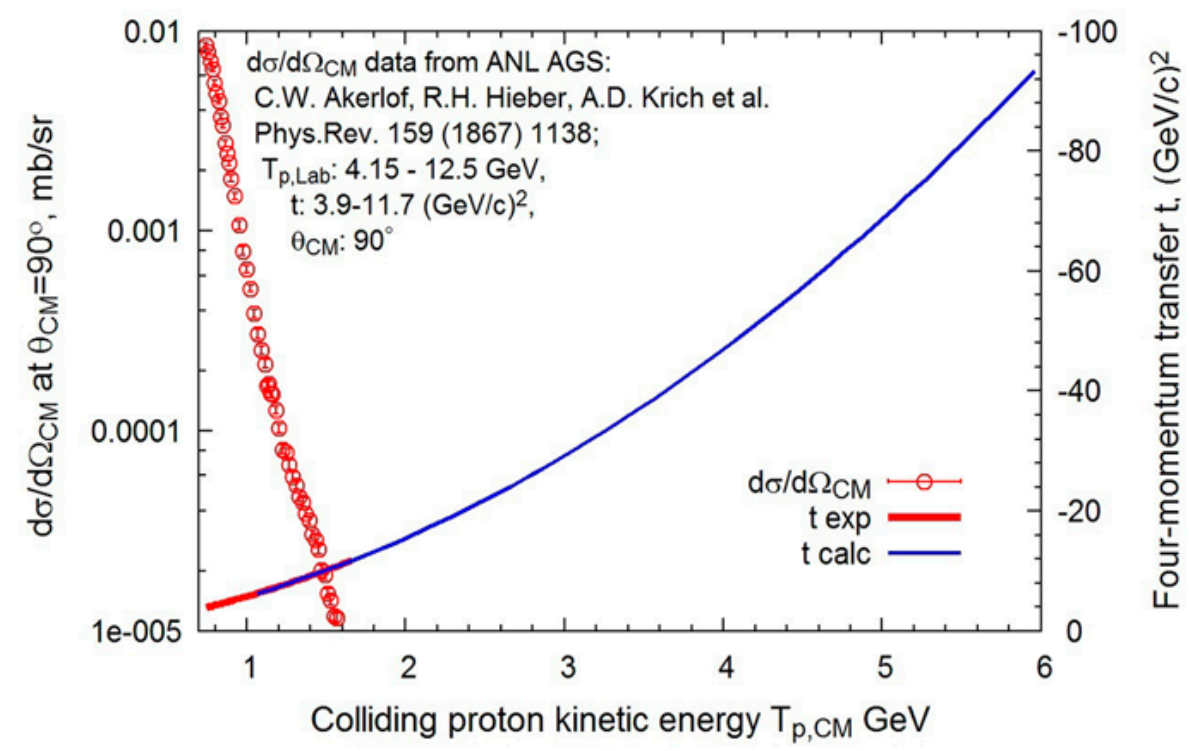

Figure 7: CM differential cross sections [एष] and 4-momentum transfer $t$ at $\theta_{C M}=90^{\circ}$ for pp collisions versus colliding nucleon energy $T_{p C M}$.

For example, let us consider the experimental data [18] in Fig. प of the measurements of the differential proton-proton cross sections $d \sigma / d \Omega_{C M}(p p)$ at the angle $\theta_{C M}=90^{\circ}$ in the center of mass and the advantage of the $C M$ kinematics. In Fig. $\square$ on the left scale there are presented $d \sigma / d \Omega_{C M}$ results at the angle $\theta_{C M}=90^{\circ}$, which were obtained at the Argonne National Laboratory Zero Gradient Synchrotron ( $A N L Z G S$ ) [1]8] in the energy region of the proton beam from $T_{p L a b}=$ $4.15 \mathrm{GeV}$ to $T_{p L a b}=12.5 \mathrm{GeV}$. This $T_{p L a b}$ energy region corresponds to the proton energies in $C M$ from $T_{p C M}=0.74 \mathrm{GeV}$ to $T_{p C M}=1.66 \mathrm{GeV}$ and the values of the 4-momentum transfer squared $t_{1,3}$ from -3.9 to $-11.7(\mathrm{GeV} / \mathrm{c})^{2}$. This $t_{1,3}$ region is shown in Fig. $\square$ by the thick red curve on the right scale. On the same scale of Fig. $\square$ the available $t_{1,3}$ values in the collider NICA energy range $T_{p C M}$ are shown by the blue curve.

\section{Experimental conditions at the polarized colliding beams of the NICA collider.}

The usage of the colliding beams of polarized nucleon for the considered measurements has a number of significant preferences in comparison with the "fixed" target experiments. At first, the angular acceptance of the collider detectors stretches over the full solid angle $4 \pi \mathrm{rad}$. Further,the wider ranges of the investigated energies of nucleons $T_{N L a b}$ and square four-momentum transfer $t$ are available (see the previous section). And, finally, the so-called "target" in such measurements does not contain background impurities, which complicate the processing of the accumulated data.

At the NICA accelerator complex [ []] (see Fig. [8]), currently under construction, it is planned to have the colliding beams of unpolarized and longitudinally and transverse polarized protons and deuterons [ㅁ, [2]]. The range of the kinetic energy of the colliding beam of polarized protons $p \uparrow p \uparrow$ is expected to be $T_{p p}=5 \div 12.6 \mathrm{GeV}\left(\sqrt{s_{p p}}=12 \div 27 \mathrm{GeV}\right)$. The range of the kinetic energy of the colliding beams of polarized deuterons $d \uparrow d \uparrow$ is expected to be $T_{d d}=2 \div 5.5 \mathrm{GeV} / u$ $\left(\sqrt{s_{N N}}=4 \div 13 \mathrm{GeV}\right)$. The polarized proton number per bunch: $\sim 1 \times 10^{12} p \uparrow$. The number of bunches in the two NICA rings: 22 . The luminosity value: $L=1 \times 10^{32} \mathrm{~cm}^{-2} \mathrm{~s}^{-1}$ at $\sqrt{s_{p p}} \geq$ $27 \mathrm{GeV}$. The estimated luminosity life time: $T_{L l f} \simeq 5.6$ hours [20]. As an example, for the RHIC polarized proton beams the luminosity life time (store) was typically six hours [22]. 


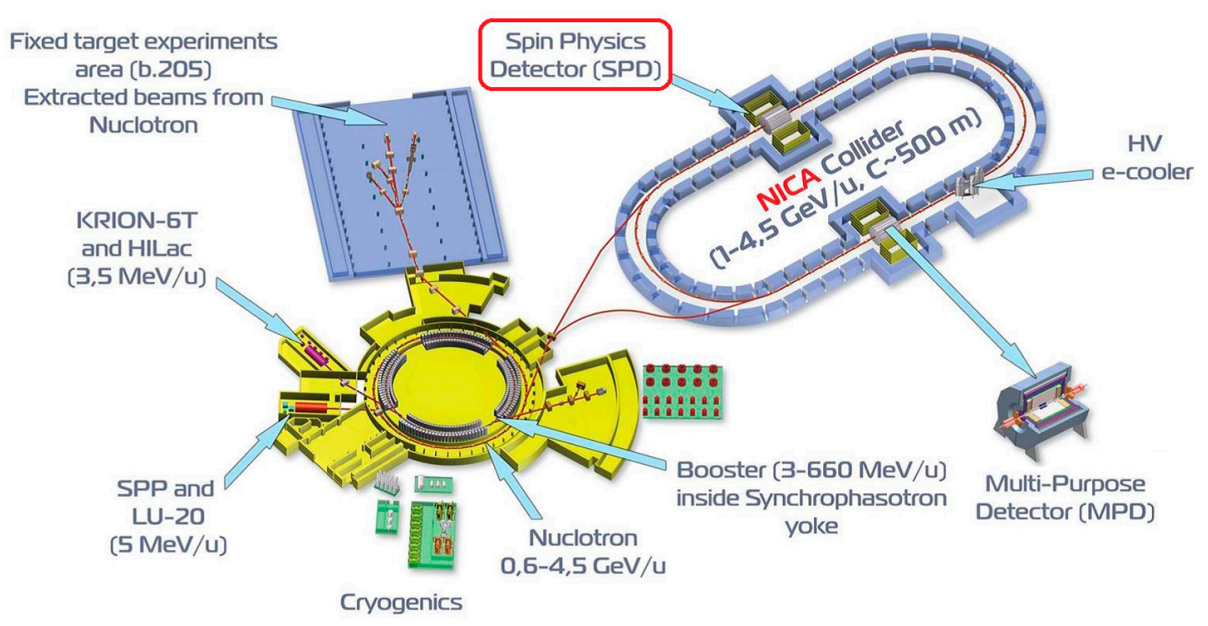

Figure 8: The NICA accelerator complex layout.

In the elastic $p \uparrow p \uparrow$ interactions in the colliding beams of polarized protons the spin-dependent $N N$ observables for isospin $I=1$ can be measured (see section $\mathbb{Z}$ ). In the case of the $(d \uparrow d \uparrow)$ collisions (particles with spin 1) one must use the appropriate formalism to determine the corresponding spin observables. The formalism describing the cases of the elastic scattering of particles with spins $\left(\frac{1}{2}, \frac{1}{2}\right),\left(1, \frac{1}{2}\right)$, and $(1,1)$ was discussed in detail in [2]]. Using such formalism similar spin-dependent observables for the quasi-elastic $N N, N d$, and $d d$ processes can be investigated. Assuming the deuteron is a loosely coupled system, one can apply the formalism of section $\square$ to describe the quasi-elastic $N N$ scattering of individual nucleons in the deuteron. Using this formal-

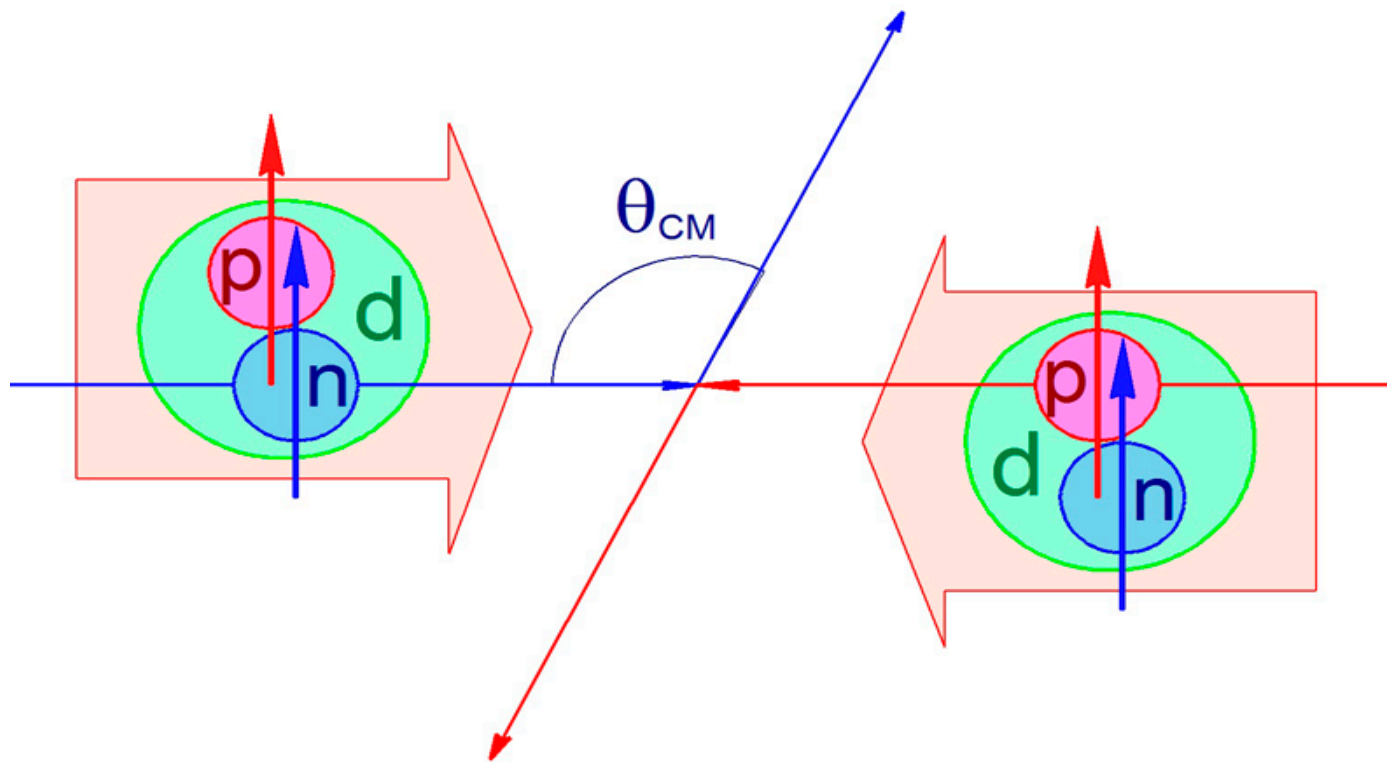

Figure 9: Schematic view of the quasi-elastic $N N$ scattering in the colliding beams of polarized deuterons.

ism in the polarized deuteron $d \uparrow d \uparrow$ collisions (see Fig. . $($ ) both $p p(I=1)$ and $n p(I=1$ and $I=0)$ quasi-elastic $A_{\text {ooio }}$ and $A_{\text {ooik }}$ observables can be measured in wide ranges of the colliding nucleon energy $E_{N C M}$ and four-momentum transfer squared $t_{1,3}$. The nucleons in the deuteron have the same value and orientation of polarization as deuteron ones $P \uparrow_{N}=P \uparrow_{d}$ and half deuteron kinetic 
energy $T_{N}=\frac{1}{2} T_{d}$.

To measure the elastic $N N$ differential cross section we have to select the elastic $N N$ events among all the inelastic ones. For the elastic $N N$ interaction in the centre-of-mass system the outgoing nucleons have the following kinematical characteristics:

1. These nucleons have equal momentum values and opposite momentum directions.

2. The absolute values of outgoing nucleon momenta are the same as for the colliding nucleon ones and do not depend on the nucleon scattering angle $\theta_{C M}$.

Such parameters of outgoing nucleons formulate the requirements for the Spin Physics Detector $(S P D)$ for the elastic events selection. The $S P D$ should provide the energy and tracks (momentum) measurements of the scattered nucleons, as well as the determination of the interaction point of the primary colliding particles.

The relative monitoring of the obtained angular distributions of the elastic $N N$ scattering events can be made by using information (readings) from the pickup electrodes placed at both sides of the intersection beams point. Since the measured value of the asymmetry is the ratio of difference and sum of the elastic event yields, measured in the same data taking run, the absolute beams intensity monitoring is not required.

For the measurement and continuously monitoring of the values and signs of the colliding beam polarizations sufficiently complete and reliable polarimetry system is needed. For this reason, it is desirable to have a number of polarimeters at several places along the polarized beam preparation line and at each of accumulated polarized beams inside the collider. The beam line polarimeters have to be placed:

1. Low energy polarimeters - before polarized beam injection into the Nuclotron.

2. Polarimeters inside the Nuclotron ring (using internal target station).

3. Special devices (polarimeters) placed at each of accumulated colliding polarized beams for the beam polarization measurement.

4. Detection by the NICA detectors of the elastic $N N$ scattering events will provide the opportunity to determine the left-right asymmetries $\mathbf{a}_{N N}$ of the reaction yields. This will enable us to estimate the polarization values of the colliding beams directly at the interaction point. This could be done both for proton and deuteron colliding beams.

When performing the relative measurements of the spin-dependent $N N$ observables it is extremely important to avoid the systematic errors of the measurement results due to the long-term drifts in the measuring conditions. It is possible to minimize the influence of such instabilities on the measurement results by the continuous change of the polarization sign of the colliding particle bunches. For this purpose it is necessary to retain the sign of the polarization of the particles (" + "or" - " or "O") at each bunch starting with the injection of the particles into the Nuclotron from the polarized ion source up to the interaction of the colliding beams at the points of intersection of the collider. The bunches should be loaded in the collider rings with various patterns of the alternating polarization sign. See for example ref's [8, [2] to the RHIC colliding $p \uparrow p \uparrow$ beams.

In the first measurements [[]] of the asymmetry $\mathbf{a}_{p p}$ in the elastic $p \uparrow p \uparrow$ scattering of the $100 \mathrm{GeV}$ protons at $R H I C$ collider, the following sequence of the sign of the polarization of the particles in the colliding bunch was used. The bunches were loaded with different patterns of alternating polarization sign, with three bunches with zero polarization, so that the collisions at the experiments included the combinations $(++,+-,-+,--, 00)$ where the signs refer to (blue,yellow accumulative rings) polarization sign for the colliding bunches. The polarization direction for each bunch was selected at the polarized $H^{-}$source, and each $R H I C$ ring was loaded with 55 bunches each with a selected spin direction, spaced $212 \mathrm{nsec}$ apart and with 5 consecutive missing bunches, used to abort the beams (the abort gap) [22]. "The bunch arrangements have changed from year 
to year, generally different in the two beams ("yellow" and "blue") to give changing combinations at each intersection region. For example, one beam might have $(++----++)$ and the other $(+-+--+-+)$. Generally the repeating pattern has been after 8 bunches." [[23].

Created at the VBLHEP accelerator complex the source of polarized ions of deuterium and hydrogen [24, 25] will provide the opportunities for the necessary sequence of the polarization signs of particles in the bunches which are loaded into both rings of the NICA collider.

\section{Estimations of the elastic event yields for measurement of the spin-dependent NN values at the NICA collider.}

According to the formulas ([2.] ) and (‥3) the spin-dependent $N N$ observables are calculated basing on the measurements of differential cross sections $d \sigma / d \Omega^{o}(E, \theta)$ and $d \sigma / d \Omega^{p o l}(E, \theta)$ in the unpolarized and polarized nucleon collisions at given values of the colliding nucleon energy $E_{N}$ and scattering angle $\theta_{N}$ in the corresponding coordinate system. To find the values $A_{\text {ooio }}\left(E_{C M}, \theta_{C M}\right)$ or $A_{\text {oooi }}\left(E_{C M}, \theta_{C M}\right)$ or $A_{\text {ooik }}\left(E_{C M}, \theta_{C M}\right)$ we need to measure in the collider measurements the differential cross sections $d \sigma / d \Omega^{o}\left(E_{C M}, \theta_{C M}\right)$ and $d \sigma / d \Omega^{p o l}\left(E_{C M}, \theta_{C M}\right)$ for the elastic $N N$ and $N \uparrow N \uparrow$ interactions at the given values of the colliding nucleon energy $E_{N C M}$ and scattering angle $\theta_{N C M}$ in the centre-of-mass.

In figures $\square$ and $\square$ we present a part of the existing data on the differential proton-proton and the neutron-proton cross sections in the center of mass system of the colliding nucleon from compilation [26]. This data set provides an opportunity to obtain reasonable estimations of the

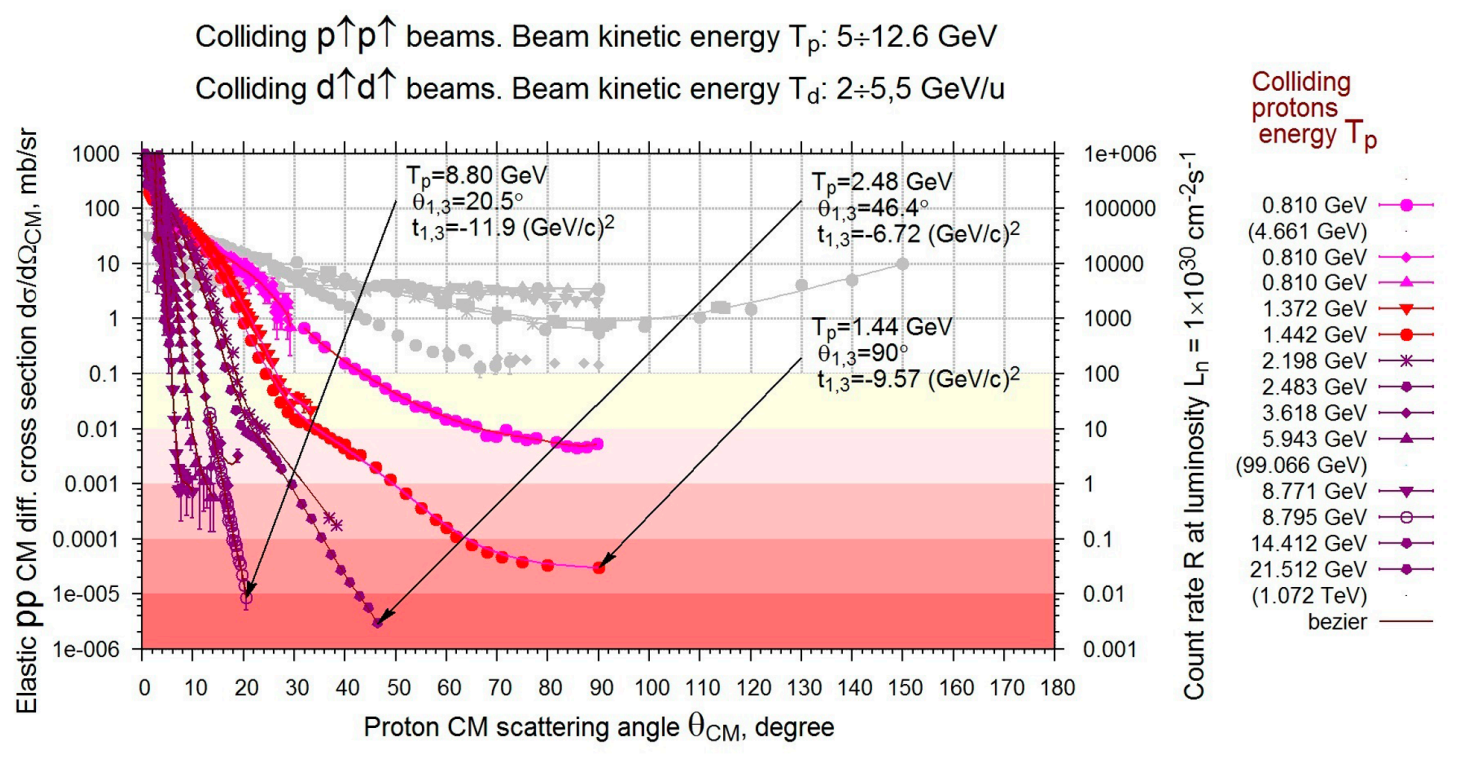

Figure 10: Experimental data set [26] on the differential $C M$ cross sections for the elastic $p p$ collisions.

outputs of the elastic $p p$ and $n p$ scattering at some of the CM scattering angles of nucleons in the whole energy range of the colliding NN beams at the NICA collider.

Figure [0] shows the set of experimental data [26] on the differential $p p$ cross sections for scattering angles from 0 to $180^{\circ}$ in a wide energy range of the interacting protons. The $x$-axis represents the scattering angle of the proton $\theta_{p C M}$ in the centre-of-mass of the colliding protons. The left logarithmic scale represents values of the differential cross sections $d \sigma / d \Omega_{C M}(p p)$ in 
the centre-of-mass of two colliding protons. The legend indicating the symbols representing the $d \sigma / d \Omega_{C M}(p p)$ values obtained at a given kinetic energy $T_{p C M}$ of the colliding protons is shown on the right edge of the figure $\mathbb{}$. The $d \sigma / d \Omega_{C M}(p p)$ data at $C M$ energies belonging to the energy range of the colliding polarized proton beams of the NICA collider is specified by dark characters. The presented $p p$ data sets are approximated with the Bezier curves of degree $\mathrm{n}$ (the number of data points) that connect the endpoints.

Experimental data [26] on the differential $n p$ cross-sections for scattering angles from 0 to $180^{\circ}$ in a wide range of energies of the interacting nucleons is shown in Fig. $\square$. The scales and symbols in this figure are similar to the corresponding values of Fig.

The event rate $R$ in the collider is proportional to the interaction cross section $\sigma_{\text {int }}$ and luminosity $L: R=\sigma_{\text {int }} \times L$. Right logarithmic scale in figures $\mathbb{}$. 0 and $\mathbb{1}$ shows the values of the count rate $R$ of the elastic $N N$ events expected for appropriate values of the cross sections $d \sigma / d \Omega_{C M}(N N)$ when the planned luminosity of the colliding polarized nucleon beams is equal to $L=1 \times 10^{30} \mathrm{~cm}^{-2} \mathrm{~s}^{-1}$.

Thus, using the known values of the differential $N N$ cross sections for specific values of the colliding nucleon energy $T_{N C M}$ and nucleon scattering angle $\theta_{N C M}$ on this scale one can estimate the expected counting rate $R$ of the elastic $N N$ scattering events. The kinematic characteristics of the boundary points of multiple data sets are shown in Figures $\square$ and $\square$ by the arrows. There the values of the nucleon kinetic energy $T_{N C M}$, the nucleon scattering angle $\theta_{N C M}$, and the squared four-momentum transfer $t_{1,3}$ are specified. These data will facilitate the estimations of the outputs of the elastic $p p$ and $n p$ processes in the center-of-mass of the interacting nucleons.
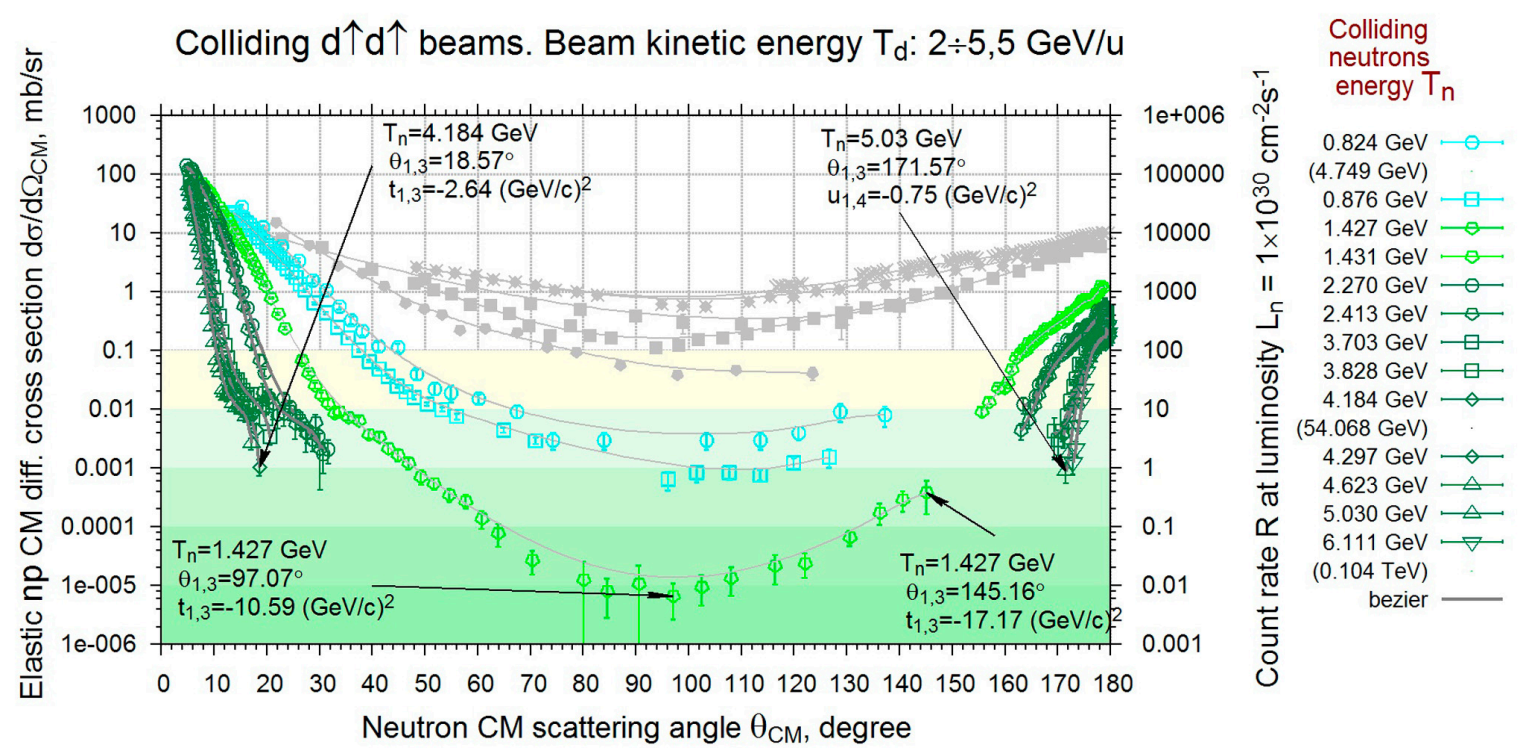

Figure 11: Experimental data set [26] on the differential $C M$ cross sections for the elastic $n p$ collisions.

For example, for a value of the cross section $d \sigma / d \Omega_{C M}(p p) \sim 1 \cdot 10^{-5} \mathrm{mb} / \mathrm{sr}$ in the left lower data endpoint in Fig. $\mathbf{U}$ with parameters $T_{p C M}=8.8 \mathrm{GeV}, \theta_{p C M}=20.5^{\circ}$, and $t_{1,3}=$ $-11.9(\mathrm{GeV} / \mathrm{c})^{2}$, the expected output of the elastic $p p$ events will be $\sim 0.01 \mathrm{event} \cdot \mathrm{sr} r^{-1} \cdot \mathrm{s}^{-1}$. The differential cross section value $d \sigma / d \Omega_{C M}(n p) \sim 1 \cdot 10^{-3} \mathrm{mb} / \mathrm{sr}$ in the left lower data endpoint in Fig. W with parameters $T_{n} C M=4.18 \mathrm{GeV}, \theta_{n C M}=18.57^{\circ}$, and $t_{1,3}=-2.64(\mathrm{GeV} / \mathrm{c})^{2}$ corresponds to the expected output of the elastic $n p$ event $\sim 1$ event $\cdot s r^{-1} \cdot s^{-1}$. The same value 
of the expected output of the elastic $n p$ event will be for differential cross section value in the right bottom data endpoint in Fig. $\square$ with parameters $T_{n C M}=5.03 \mathrm{GeV}, \theta_{n} \mathrm{CM}=171.57^{\circ}$, and $u_{1,4}=-0.75(\mathrm{GeV} / \mathrm{c})^{2}$.

We have also made a comparison of the outputs of the elastic $N N$ events in the collider experiments with the experiments on nucleon beam with the "fixed" target. The planned luminosity in the spin experiments at the NICA collider is estimated by a value of $L=1 \times 10^{30} \mathrm{sm}^{-2} \mathrm{~s}^{-1}$ for the polarized $p \uparrow p \uparrow$ colliding beams. Thus one count per second will correspond to the events with total cross section of $\sigma_{t o t} \sim 10^{30} \mathrm{sm}^{-2}(1 \mu \mathrm{b})$. In the experiments with "fixed" targets with the use of a polarized proton target $\left(n_{H} \sim 10^{24} \mathrm{~cm}^{-2}\right)$ and polarized proton beam $I \sim 10^{10} \mathrm{p} \uparrow /$ cycle, one count per second will correspond to the events with total cross section of $\sigma_{t o t} \sim 10^{-34} \mathrm{sm}^{-2}(100 \mathrm{pb})$.

\section{Conclusion.}

The possibilities of measuring the spin-dependent $N N$ observables in the elastic $p p$ collisions and quasi-elastic $N N$ interactions of quasi-free nucleons in the deuteron at the colliding $p \uparrow p \uparrow$ and $d \uparrow d \uparrow$ beams of the NICA collider, currently under construction at VBLHEP JINR, were considered in the present report.

There was briefly considered a $S$-matrix formalism [[123, [4, [5] that specifies the spin-dependent observables in the elastic nucleon-nucleon scattering. It is shown what spin-dependent $N N$ observables can be measured using detectors for the collider and what scattering characteristics should be measured to determine the spin-dependent $N N$ values.

The kinematic characteristics of the $N N$ scattering processes at the laboratory system and in the center of mass system of the colliding nucleons were analyzed. It is shown that in the elastic $N N$ scattering at the colliding beams the broader range of the energy values of the interacting nucleons and the square of the four-momentum transfer are achieved in comparison with the experiment used the "fixed" target. Other significant advantages of the measurements at the collider compared with the experiments with the "fixed" targets are the following: the angular acceptance of the collider detector extends to the full solid angle $4 \pi \mathrm{rad}$ and the so-called "target" in such measurements does not contain background impurities, which complicate the processing of the accumulated data.

The expected parameters of the polarized colliding $p \uparrow p \uparrow$ and $d \uparrow d \uparrow$ beams of the NICA collider under construction are listed. The requirements for the collider detectors for registration and selection of the elastic $N N$ events and to polarimetry system for the prepared polarized colliding beams were formulated. The organization of loading of the particle bunches in the collider rings by different samples of alternative signs of the particle polarization was also mentioned.

The existing data sets from compilation [26] on the differential elastic $p p$ and $n p$ cross sections in the center-of-mass of the colliding nucleons for the expected range of nucleon energies were shown. The output estimations of the elastic $N N$ events were presented and the comparison of the collider measurements with the experiments in the laboratory system using particle beam with the "fixed" target were made.

The data planned to be obtained in the measurements of the elastic $N N$ scattering observables will promote a creation of an adequate phenomenological and theoretical description of the $N N$ interaction over energy region of $\sqrt{s_{N N}}=4 \div 27 \mathrm{GeV}$.

\section{Acknowledgements}

The author thanks H.M. Spinka for providing the necessary information.

The author is particularly grateful to his colleague E.V. Chernykh for the help during the talk and this contribution preparing. 


\section{References}

[1] Conceptual project Design and construction of Nuclotron-based Ion Collider fAcility (NICA) and Mixed Phase Detector (MPD)", NICA - project, Dubna, JINR P9-2008-153, 2008, 51 pages.

[2] NICA - Nuclotron - based Ion Collider fAcility - a Base for Fundavental Research and Innovation Development, JINR, Dubna 2014, http://nica.jinr.ru/files/Booklet-NICA.pdf

[3] Ruprecht Machleidt, Professor of Physics, University of Idaho. Private communication.

[4] R. A. Arndt, W. J. Briscoe, I. I. Strakovsky, and R. L. Workman, Updated analysis of NN elastic scattering to $3 \mathrm{GeV}$., Phys. Rev. C 76, 2007, p. 025209. [arXiv:0706.2195 [nucl-th]].

[5] A. Krisch, Hard Collisions of Spinning Protons: History \& Future, Invited talk at DSPIN-09, 1-5 September 2009, JINR, Dubna, Proceedings, E1,2-2010-13, JINR, Dubna 2010.

[6] C. Lechanoine-LeLuc and F. Lehar, Nucleon-nucleon elastic scattering and total cross sections, Reviews of Modern Physics v. 65, No. 1, 1993, p. 47.

[7] A. Krisch, Polarized Beams and Violent Collisions of Spinning Protons: Past, Present\& Future, in proceedings of XV Advanced Research Workshop on High Energy Spin Physics (DSPIN-13),Dubna, October 8-12, 2013 [http://theor.jinr.ru/ spin/2013/talks/krisch.pdf].

[8] G. Bunce, The First RHIC Spin Run at PHENIX and PP2PP, arXiv:hep-ex/0208002v1, 31 Jul 2002.

[9] S. Bültmann et al., First measurement of proton-proton elastic scattering at RHIC, Physics Letters, B 579, 2004, p. 245.

[10] A. Sandacz, Results from PP2PP Experiment at RHIC, arXiv:hep-ex/0510032v2, 17 Nov 2005.

[11] S. Bültmann et al., Double Spin Asymmetries $A_{\text {oonn }}$ and $A_{\text {ooss }}$ at $\sqrt{s}=200 \mathrm{GeV}$ in Polarized Proton-Proton Elastic Scattering at 4RHIC4, arXiv:nucl-ex/0610022v2, 6 Mar 2007.

[12] I.Savin, Spin Physics Experiments @ NICA-SPD with polarized proton and deuteron beams. Letter of Intent, talk at Workshop NICA-SPIN-2014, Prague, February 10-16.

[13] J. Bystricky, F. Lehar, and P. Winternitz, Formalism of nucleon-nucleon elastic scattering experiments, J. Phys. (Paris). v. 39, 1978, p. 1.

[14] S.M. Bilenky, L.I. Lapidus, and R.M. Ryndin, Polarized proton target in experiments with high-energy particles, Usp. Fiz. Nauk, v. 84, 1964, p. 243.

[15] L.D. Puzikov, R.M. Ryndin, and Ya.A. Smorodinskii, Reconstruction of the Scattering Matrix of Two-Nuclton System. Zh. Eksp.Teor. Fiz., v. 32, 1957, p. 592.

[16] V.I, Sharov et al., Measurement of the $n p$ total cross section difference $\Delta \sigma_{L}(n p)$ at $1.39 \mathrm{GeV}$, $1.69 \mathrm{GeV}, 1.89 \mathrm{GeV}$ and $1.99 \mathrm{GeV}$. European Physical Journal C37, 2004, p. 79, [Physics of Atomic Nuclei, Vol. 68, No. 11, 2005, pp. 1796-1811. From Yadernaya Fizika, Vol. 68, No. 11, 2005, pp. 1858-1873.]

[17] D.P. Grosnick et al., Measurement of the differences in the total cross section for antiparallel and parallel longitudinal spins and a measurement of parity nonconservation with incident polarized protons and antiprotons at $200 \mathrm{GeV} /$ c., Phys. Rev. D 55, 1997, p. 1159.

[18] C.W. Akerlof, R.H. Heiber, A.D. Krish, K.W. Edwards, L.G. Ratner, and K. Ruddick, Eiastic Proton-Proton Scattering at $90^{\circ}$ and Structure within the Proton, Phys.Rev. 159, No. 5, 1967, p. 1138.

[19] I.N. Meshkov, Talk: Status of NICA Project, 23 - 29 August 2010, JINR, Dubna.

[20] A.D. Kovalenko, Polarized deuterons \& protons at NICA: Status of the Concept Optimization, Talk at Workshop NICA-SPIN-PRAGUE 2014, Charles University, Prague 10-15 February, 2014. 
[21] G.G. Ohlsen, Polarization transfer and spin correlation experiments in nuclear physics., Rep. Prog. Phys. 35, 1972, p.p. 717-801.

[22] I. G. Alekseev et al., Measurements of single and double spin asymmetry in pp elastic scattering in the CNI region with a polarized atomic hydrogen gas jet target, Phys.Rev. D 79, 2009, p. 094014.

[23] H.M. Spinka, ANL, IL, Private communication.

[24] V.V. Fimushkin, A.S.Belov, A.D.Kovalenko et al. EPJ, Special Topics, 162, 2008, pp. 275-280.

[25] V.Fimushkin, A.D. Kovalenko et al. Status of the Polarized Ions Source for the JINR Accelerator Complex Physics of Part.\& Nucl., 2014, Vol.45, No.1, 2014, pp. 297-300, Springer.

[26] J. Bystricky and F. Lehar, Nucleon-Nucleon Scattering Data, editors H. Behrens and G. Ebel, Fachinformationszentrum Karlsruhe, 1981 Edition, Nr. 11-2 and Nr. 11-3, 1981. 